\title{
Study on dynamic characteristics of gas films of spherical spiral groove hybrid gas bearings
}

\author{
Chenhui Jia ${ }^{1}$, Haijiang Zhang ${ }^{1}$, Shijun Guo ${ }^{2}$, Ming Qiu ${ }^{1}$, Wensuo Ma ${ }^{1,3}$, Zhuangya Zhang ${ }^{1}$ \\ (1.School of Mechatronics Engineering, Henan University of Science and Technology, \\ Luoyang, Henan 471003, \\ 2. School of Aerospace, Transport and Manufacturing, Cranfield University, England, \\ MK43 0AL, \\ 3. Machinery and equipment advanced manufacturing collaborative innovation center in \\ Henan Province, China)
}

\begin{abstract}
According to the gas film force variation law, when the bearing axis is slightly displaced from the static equilibrium position, displacement and velocity disturbance relation expressions for the gas film force increment are constructed. Moreover, combined with the bearing rotor system motion equation, calculation model equations for the gas film stiffness and damping coefficients are established. The axial and radial vibration and velocity of the gas bearings during operation are collected. The instantaneous stiffness and damping coefficients of the gas film are calculated by the rolling iteration algorithm using MATLAB. The dynamic changes in the gas film stiffness and damping under different motion states are analysed, and the mechanism of the gas film vortex and oscillation is studied. The results demonstrate the following. (1) When the gas bearing is running in the linear steady state in cycle 1, the dynamic pressure effect is enhanced and the stability is improved by increasing the eccentricity; when the gas supply pressure is increased, the static pressure effect is enhanced and the gas film vortex is reduced, but the oscillation is strengthened. (2) With the increase in rotational speed, the gas film vortex force gradually exceeds the gas film damping force, and the stability gradually worsens, causing a fluctuation in the gas film stiffness and damping, following which singularity occurs and a half-speed vortex is formed. Meanwhile, the gas film oscillation is intensified, and the rotor enters the nonlinear stable cycle 2 state operation. (3) As the fluctuation of the film force increases, the instantaneous stiffness and damping oscillation of the film intensifies, most of the stiffness and damping coefficients exhibit distortion, and the rotor operation will enter a chaotic or unstable state. Therefore, the gas bearing stiffness and damping variation characteristics can be used to study and predict the gas bearing operating state. Finally, measures for reducing the vortex and oscillation of the gas film and improving the stability of the gas bearing operation are proposed.
\end{abstract}

Key words: Spherical hybrid gas bearings; Stiffness damping model equation of gas film; Parameter data acquisition; Dynamic characteristic analysis; Gas film vortex and oscillation; Nonlinear stability

\section{Introduction}

Hybrid gas bearings display the characteristics of high rotational speed, high precision, and stable operation, as well as being frictionless ${ }^{(1-3)}$. They play an irreplaceable role in the fields of aerospace, micro-engineering, precision instruments, medical equipment, and high-end equipment ${ }^{(4-6)}$.

Dynamic and static pressure exist simultaneously in hybrid gas bearings and are mutually coupled; the dynamic characteristics of a gas film are extremely complex. Owing to the compressibility of the gas, the dynamic pressure effect occurs when the rotor is 
operating at a high speed, and a gas film vortex occurs. Meanwhile, the power frequency vibration caused by the rotor imbalance, and the interaction of self-excited vibration and gas film vortex will cause gas film oscillation, which will lead to rubbing of the bearing rotor and bearing instability ${ }^{(7) 8)}$. Therefore, it is of great significance to study the dynamic characteristics of gas bearings, reduce the gas film vortex and oscillation, improve the stability of gas bearings, and further improve the bearing capacity and stability of bearings ${ }^{(9)}$.

At present, domestic and foreign scholars have conducted a significant amount of theoretical research on the nonlinear stability and dynamic characteristics of gas bearings $\mathrm{s}^{(10 \text {, }}$ 11). Owing to the assumptions of the lubrication theory model and simplification of the calculation model, the theoretical calculation method cannot accurately reflect the nonlinear coupling relationship between the dynamic pressure and static pressure diffusion effects of gas bearings ${ }^{(12-14)}$. The combination of theoretical calculations and experimental tests is also scarce in the research, which results in the calculation of the dynamic bearing capacity and stiffness damping of the gas film deviating from the true value, and therefore large errors ${ }^{(11,15,16)}$.

This paper is based on the mathematical model of lubrication analysis for gas bearings. Equations are established for the gas film stiffness and damping coefficients. Combined with experimental data, the dynamic variations in the film stiffness and damping under different motion conditions are analysed, and the mechanism of the gas film vortex and oscillation is studied. The purpose of this study is to improve the dynamic characteristics of gas bearings, reduce the gas film vortex and oscillation, and improve the gas bearing operation stability.

\section{Calculation and analysis model for dynamic characteristics of spherical spiral groove hybrid gas bearings}

\subsection{Mathematical model of spherical spiral groove dynamic-static pressure gas bearing}

Figure 1 presents a schematic of spherical spiral groove hybrid gas bearings, where $\beta$ is the spiral angle; $\omega$ is the rotational speed; $\varphi$ is the gas supply tangential angle; $\alpha_{0}$ is the small end angle of the rotor; $\alpha_{1}$ is the initial angle of the spiral groove; $\alpha_{2}$ is the large end angle of the rotor; $p_{s}$ is the supply pressure; $b_{r}$ is the table width; $b_{g}$ is the groove width; $h_{g}$ is the film thickness at a groove; and $h_{0}$ is the average gas film thickness.

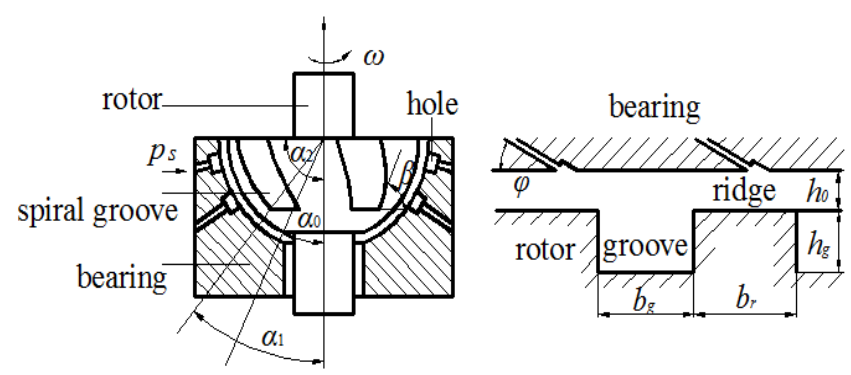

Figure 1. Schematic diagram of spherical gas bearings 


\subsection{Mechanical model of gas film}

When the rotor of a gas bearing is operating, the rotor deviates from the steady equilibrium position and moves around $\mathrm{it}^{(17)}$. The relation expression for the displacement and velocity disturbances and the increment in the gas film force is expressed, and combined with the bearing rotor, the system motion equation calculates the stiffness and damping coefficients of the gas film. When a bearing rotor is disturbed in a static equilibrium position, the rotor deviates from the static equilibrium position in the $\Phi, \xi$, and $z$ directions, and carries out micro-motion. Its force is illustrated in Figure 2.
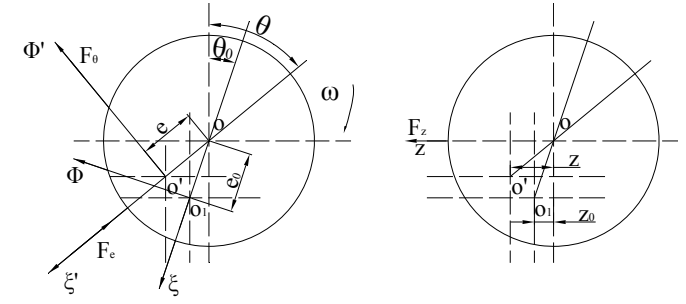

Figure 2. Rotor dynamic force diagram

The $P$ value that represents the gas film pressure distribution of the rotor axis should be the function expression for the instantaneous position of the rotor axis $(e, \xi$, and $z)$ and instantaneous displacement velocity $\left(V_{e}, V_{\xi}\right.$, and $\left.V_{z}\right)$ as the parameter.

The gas film force of the axis in the static equilibrium position can be expressed as

$$
p_{0}=p\left(\xi, \phi, z ; e_{0}, \theta_{0}, z_{0}, 0,0,0\right)
$$

The pressure distribution of the gas film during rotor displacement motion can be expressed as

$$
p_{0}=p(\xi, \phi, z ; e, \theta, z, \dot{e}, e \dot{\theta}, \dot{z})
$$

The gas film pressure distribution $P$ is expanded into a Taylor series of instantaneous displacement and displacement velocity when the rotor deviates from the steady equilibrium position. Moreover, because the bearing rotor amplitude and gas film thickness are small, and the rotor moves slightly in the static equilibrium position, and minor terms greater than second order will be omitted.

$$
p=p_{0}+p_{e} \Delta e+p_{\theta}(\mathrm{e} \Delta \theta)+p_{z} \Delta z+p_{\dot{e}} \Delta \dot{e}+p_{\dot{\theta}} e \Delta \dot{\theta}+p_{\dot{z}} \Delta \dot{z}
$$

where $p_{e}, p_{\theta}, p_{z}, p_{\dot{e}}, p_{\dot{\theta}}, p_{\dot{z}}$ denotes the perturbation pressure of the distribution $P$ varying with $\Delta e, \mathrm{e} \Delta \theta, \Delta z, \dot{e}, \mathrm{e} \dot{\theta}, \dot{z}$.

The axial and radial integration by means of Equation (3) provides the gas film force during slight movement of the rotor:

$$
\left.\begin{array}{l}
F_{e} \\
F_{\theta} \\
F_{z}
\end{array}\right\}=-\left\{\begin{array}{l}
R_{0}^{2} \int_{\alpha_{2}}^{\alpha_{0}} \int_{0}^{2 \pi} p \cos \phi \sin ^{2} \alpha d \phi d \alpha \\
R_{0}^{2} \int_{\alpha_{2}}^{\alpha_{0}} \int_{0}^{2 \pi} p \sin \phi \sin ^{2} \alpha d \phi d \alpha \\
R_{0}^{2} \int_{\alpha_{2}}^{\alpha_{0}} \int_{0}^{2 \pi} p \cos \alpha \sin \alpha d \phi d \alpha
\end{array}\right.
$$

The gas film force in the static equilibrium position of the rotor can be expressed as:

$$
\left.\begin{array}{l}
F_{e 0} \\
F_{\theta 0} \\
F_{z 0}
\end{array}\right\}=-\left\{\begin{array}{l}
F_{e}\left(e_{0}, \theta_{0}, z_{0}, 0,0,0\right) \\
F_{\theta}\left(e_{0}, \theta_{0}, z_{0}, 0,0,0\right) \\
F_{z}\left(e_{0}, \theta_{0}, z_{0}, 0,0,0\right)
\end{array}\right.
$$


The gas film stiffness and damping coefficients are partial derivatives of the gas film force to the rotor axis displacement and displacement velocity. The spherical spiral groove hybrid gas bearing has nine stiffness coefficients, $k_{i, j}$. Here, $i$ represents the direction of the film force increment, while $j$ represents the axial displacement increment direction.

$$
\begin{aligned}
& k_{e e}=\left(\frac{\partial F_{e}}{\partial e}\right)_{0} ; k_{e \theta}=\left(\frac{\partial F_{e}}{e \partial \theta}\right)_{0} ; k_{e z}=\left(\frac{\partial F_{e}}{\partial z}\right)_{0} ; k_{\theta e}=\left(\frac{\partial F_{\theta}}{\partial e}\right)_{0} ; k_{e \theta}=\left(\frac{\partial F_{\theta}}{e \partial \theta}\right)_{0} ; k_{e z}=\left(\frac{\partial F_{\theta}}{\partial z}\right)_{0} ; \\
& k_{z e}=\left(\frac{\partial F_{z}}{\partial e}\right)_{0} ; k_{z \theta}=\left(\frac{\partial F_{z}}{e \partial \theta}\right)_{0} ; k_{z z}=\left(\frac{\partial F_{z}}{\partial z}\right)_{0} ;
\end{aligned}
$$

The nine damping coefficients are represented by $b_{i, j}$, where $i$ indicates the direction of the gas film force increment, and $j$ indicates the axial velocity increment direction.

$$
\begin{aligned}
& b_{e e}=\left(\frac{\partial F_{e}}{\partial \dot{e}}\right)_{0} ; b_{e \theta}=\left(\frac{\partial F_{e}}{e \partial \dot{\theta}}\right)_{0} ; b_{e z}=\left(\frac{\partial F_{e}}{\partial \dot{z}}\right)_{0} ; b_{\theta e}=\left(\frac{\partial F_{\theta}}{\partial \dot{e}}\right)_{0} ; b_{\theta \theta}=\left(\frac{\partial F_{\theta}}{e \partial \dot{\theta}}\right)_{0} ; b_{\theta z}=\left(\frac{\partial F_{\theta}}{\partial \dot{z}}\right)_{0} ; \\
& b_{z e}=\left(\frac{\partial F_{z}}{\partial \dot{e}}\right)_{0} ; b_{z \theta}=\left(\frac{\partial F_{z}}{e \partial \dot{\theta}}\right)_{0} ; b_{z z}=\left(\frac{\partial F_{z}}{\partial \dot{z}}\right)_{0} ;
\end{aligned}
$$

When the bearing rotor axis moves slightly in a static equilibrium position, the linear increment of the gas film force is expressed as

$$
\left\{\begin{array}{l}
\Delta F_{e}=F_{e}-F_{e 0}=k_{e e} \Delta e+k_{e \theta} e \Delta \theta+k_{e z} \Delta z+b_{e e} \Delta \dot{e}+b_{e \theta} e \Delta \dot{\theta}+b_{e z} \Delta \dot{z} \\
\Delta F_{\theta}=F_{\theta}-F_{\theta 0}=k_{\theta e} \Delta e+k_{\theta \theta} e \Delta \theta+k_{\theta z} \Delta z+b_{\theta e} \Delta \dot{e}+b_{\theta \theta} e \Delta \dot{\theta}+b_{\theta z} \Delta \dot{z} \\
\Delta F_{z}=F_{z}-F_{z 0}=k_{z e} \Delta e+k_{z \theta} e \Delta \theta+k_{z z} \Delta z+b_{z e} \Delta \dot{e}+b_{z \theta} e \Delta \dot{\theta}+b_{z z} \Delta \dot{z}
\end{array}\right.
$$

When the rotor moves slightly in a static equilibrium position, the rotor centre deviates from the static equilibrium position in the $\varphi, \xi$, and $z$ directions. The instantaneous operating parameters are $\Delta e, e \Delta \theta, \Delta z, \Delta \dot{e}, e \Delta \dot{\theta}, \Delta \dot{z}, \Delta \ddot{e}, e \Delta \ddot{\theta}$, and $\Delta \ddot{z}$, and the corresponding gas film force increments are $\Delta F_{e}, \Delta F_{\theta}$, and $\Delta F_{z}$. The motion equation for the bearing rotor system is as follows:

$$
\left\{\begin{array}{l}
m \ddot{e}+\Delta F_{e}=0 \\
m e \ddot{\theta}+\Delta F_{\theta}=0 \\
m \ddot{z}+\Delta F_{z}=0
\end{array}\right.
$$

The stiffness damping model equation for the gas film can be obtained by Equations (6) and (7) simultaneously.

$$
\left\{\begin{array}{l}
m \ddot{e}+k_{e e} \Delta e+k_{e \theta} e \Delta \theta+k_{e z} \Delta z+b_{e e} \Delta \dot{e}+b_{e \theta} e \Delta \dot{\theta}+b_{e z} \Delta \dot{z}=0 \\
m e \ddot{\theta}+k_{\theta e} \Delta e+k_{\theta \theta} e \Delta \theta+k_{\theta z} \Delta z+b_{\theta e} \Delta \dot{e}+b_{\theta \theta} e \Delta \dot{\theta}+b_{\theta z} \Delta \dot{z}=0 \\
m \ddot{z}+k_{z e} \Delta e+k_{z \theta} e \Delta \theta+k_{z z} \Delta z+b_{z e} \Delta \dot{e}+b_{z \theta} e \Delta \dot{\theta}+b_{z z} \Delta \dot{z}=0
\end{array}\right.
$$

\section{Solution of equation for stiffness damping of gas film}

\subsection{Test data pre-processing method}

The initial rotor displacement is $X_{0}=Z_{0}=0, Y_{0}=0, \theta_{0}=0$, the sampling frequency is $F$, and the time interval is $\Delta t=1 / F$. When solving the gas film stiffness and damping coefficients of the model Equation (8), the constant coefficients of the model equation 
must first be obtained. Therefore, the data processing system needs to pre-process the test data of the bearing rotors $X_{n}$ and $Y_{n}$, and axial direction $Z_{n}$ vibration and rotation velocity, in the MYSQL database.

The data processing system reads the experimental data at the nth sampling bearing rotor displacement $X_{n}, Y_{n}$, and $Z_{n}$, rotational speed $V_{n}$, and sampling frequency $F$ from the MYSQL database, and performs pre-treatment according to the following formula:

$$
e_{n}=\sqrt{X_{n}^{2}+Y_{n}^{2}}, \theta_{n}=\arctan \frac{X_{n}}{Y_{n}}
$$

The rotor eccentricity $e_{n}$ and eccentric angle $\theta_{n}$ are calculated and stored; the data processing system reads the result of the previous data pre-processing from internal memory, and performs the following calculation:

$$
\Delta Z_{n}=Z_{n}-Z_{n-1}, \Delta e_{n}=e_{n}-e_{n-1}, \Delta \theta_{n}=\theta_{n}-\theta_{n-1}
$$

The variation in the $Z$-axis rotor displacement, variation in eccentricity $\Delta e_{n}$, and variation in eccentric angle $\Delta \theta_{n}$ are calculated and stored. The data processing system reads the result of the previous data pre-processing from internal memory and performs the following calculation simultaneously:

$$
\dot{e}_{n}=\frac{\Delta e}{\Delta t}, \Delta \dot{e}_{n}=\frac{\Delta e_{n}-\Delta e_{n-1}}{\Delta t}, \dot{\theta}_{n}=\frac{\Delta \theta_{n}}{\Delta t}, \Delta \dot{\theta}_{n}=\frac{\Delta \theta_{n}-\Delta \theta_{n-1}}{\Delta t}
$$

The first-order derivatives of the rotor eccentricity, eccentric angles $\dot{e}_{n}$ and $\dot{\theta}_{n}$, and variations in the first-order derivatives of the rotor eccentricity and eccentric angles $\Delta \dot{e}_{n}$ and $\Delta \dot{\theta}_{n}$ are calculated and stored. The data processing system reads the result of the previous data pre-processing from internal memory and performs the following calculation simultaneously:

$$
\ddot{e}_{n}=\frac{\dot{e}_{n}-\dot{e}_{n-1}}{\Delta t}, \ddot{\theta}_{n}=\frac{\dot{\theta}_{n}-\dot{\theta}_{n-1}}{\Delta t}, \ddot{Z}_{n}=\frac{Z_{n+1}+Z_{n-1}-2 \Delta Z_{n}}{\Delta t^{2}}
$$

The second-order derivatives of the rotor eccentricity, eccentric angles, and variation in the Z-axis rotor displacement are obtained.

At this point, the data processing system completes first-order pre-processing, and the constant coefficients $\Delta Z_{n}, \Delta e_{n}, \Delta \theta_{n}, \Delta \dot{e}_{n}, \Delta \dot{\theta}_{n}, \Delta \dot{Z}_{n}, \ddot{e}_{n}, \ddot{\theta}_{n}$ and $\ddot{Z}_{n}$ of Equation (8) at the $\mathrm{n}^{\text {th }}$ sampling of the bearing rotor are obtained.

The data processing system pre-processes the experimental data according to the above procedure, and the calculation results are stored as constant sequences according to the sampling order.

\subsection{Solution of dynamic stiffness damping of gas film}

To solve for the gas film stiffness and damping coefficients, the model described by Equation (8) must consist of six different equalities, where the determinant of the coefficient matrix is nonzero.

During the experiment, the sampling frequency of the data collection system is very high. It is considered that the rotor only undergoes minute movements during the six sampling processes, and the changes in the rotor operation state and gas film force are negligible ${ }^{(18)}$. Therefore, the pre-processed data of the first to sixth sampling in the MYSQL database are substituted into the model matrix described by Equation (9) for calculation; the instantaneous stiffness and instantaneous damping coefficients are obtained in the sixth sampling process. Thereafter, the pre-processed data of the second to seventh sampling processes in the MYSQL database are substituted into the model matrix 
of Equation (9) for calculation; the instantaneous stiffness and damping coefficients are obtained in the seventh sampling process. Following the above method, every time sampling is performed, the data processing system moves forward once in the constant coefficient sequence with equal width, and the updated data are substituted into the model matrix (Equation (9)) once again to calculate the instantaneous stiffness and instantaneous damping coefficients in that sampling process. Based on this rolling algorithm, the experimental pre-treatment data are iteratively calculated ${ }^{(19)}$. The gas bearing gas film stiffness and damping coefficients can then be obtained. Equation (9) describes the gas film stiffness and damping coefficient model matrix:

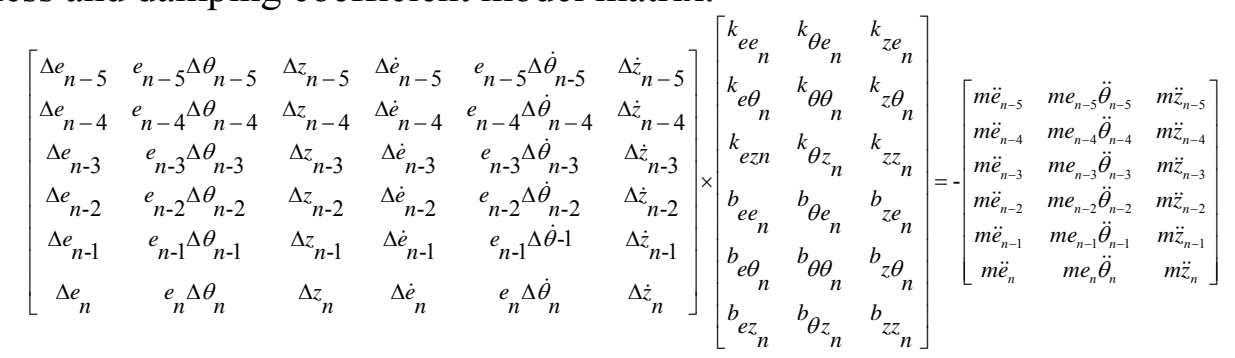

Solving the model matrix of Equation (9) according to the above process results in 18 sets of stiffness and damping coefficients for the gas film of a gas bearing, which can be obtained in one attempt.

$$
\left[\begin{array}{lll}
k_{e e_{n}} & k_{\theta e_{n}} & k_{z e_{n}} \\
k_{e \theta_{n}} & k_{\theta \theta_{n}} & k_{z \theta_{n}} \\
k_{e z n} & k_{\theta z_{n}} & k_{z z_{n}} \\
b_{e e_{n}} & b_{\theta e_{n}} & b_{z e_{n}} \\
b_{e \theta_{n}} & b_{\theta \theta_{n}} & b_{z \theta_{n}} \\
b_{n} & b_{n z_{n}} & b_{z z_{n}} \\
{ } z_{n} & b_{n} &
\end{array}\right]
$$

By means of $K_{i, j}=k_{i, j} / p_{a} R_{0}^{2}$ and $B_{i, j}=b_{i, j} h_{0} \omega /\left(p_{a} R_{0}^{2}\right)$, the form of the stiffness and damping coefficients of gas film can be converted from dimensional to dimensionless.

The MATLAB code was debugged, the data interface was set to the MYSQL database, and MATLAB data processing code was written in terms of the above method for calculation of the stiffness damping coefficients of the gas film.

\section{Test establishment and testing system}

\subsection{Test principle of stability and dynamic characteristics of gas bearing}

The experimental principle is illustrated in Figure 3. High-pressure gas source I provides high-pressure gas to bearing tester II, which suspends the rotor and provides gas power to drive the turbine and rotate the rotor. Signal detection system III measures the rotor rotational speed, displacement, and rubbing signal, and uses data processing system IV to process, calculate, and monitor the bearing rotor operating status. 


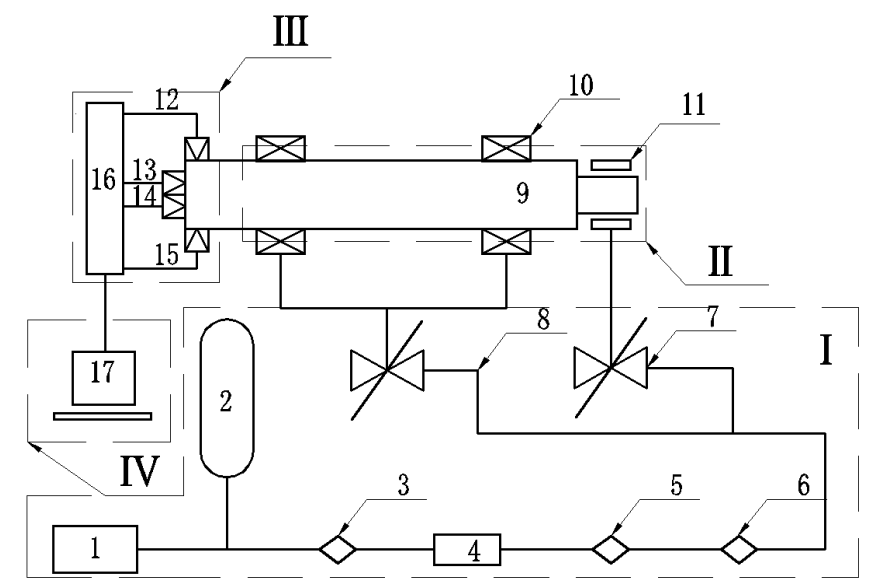

1-Gas compressor; 2-Gas storage tank; 3-High temperature freeze drier; 4-Separator filter; 5-Main pipeline filter; 6-Oil removal filter; 7-Turbine valve; 8-Bearing gas supply valve; 9-Bearing rotor; 10-Gas bearing; 11-Turbine; 12、13、14-X, Y, Z axis laser displacement sensor; 15-Speed sensor; 16-Data acquisition device; 17-Computer

Figure 3. Overall scheme diagram of test machine

\subsection{Design of main body of test machine}

The structural diagram of the bearing test machine is presented in Figure 4. The gas bearing tester primarily consists of a bearing rotor system, turbine driver, bearing seat, frame, and rubbing closed-loop circuit. Bearing rotor 3 and loading nut 9 are made of 7075 aluminium alloy, gas bearing 2 is made of graphite, and the other tester components are all made of non-magnetic stainless steel. The hemisphere diameter is $50 \mathrm{~mm}$, and the gas film thickness is $30 \mu \mathrm{m}$ in the tester bearing design; the specific parameters are listed in Table 1.
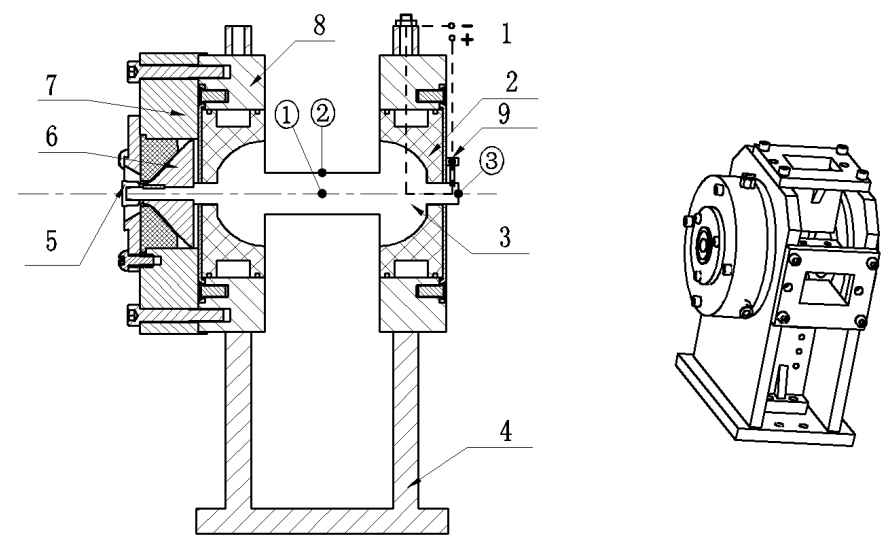

1-Connection terminal of rotor rubbing loop; 2-Gas bearing; 3-Rotor; 4-Frame; 5-Z axle load nut; 6Turbine; 7-Turbine cover; 8-Bearing chock; 9-Flexible copper brush

Figure 4. Structural chart of test machine 

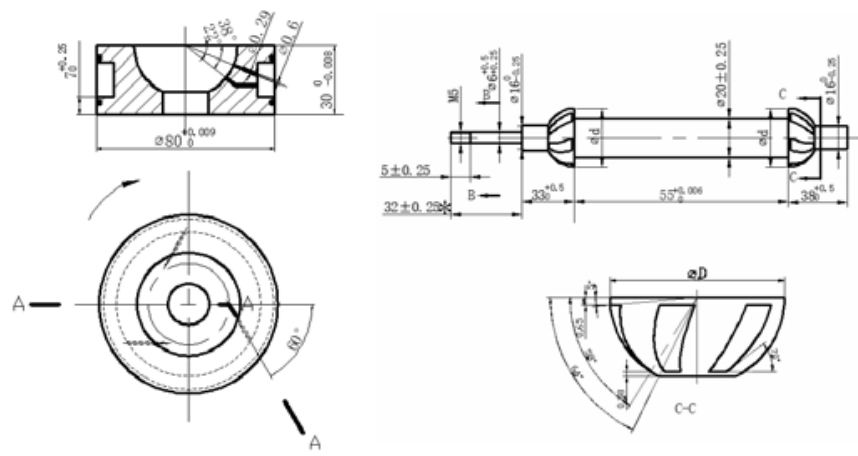

Figure 5. Test bearing and rotor parts structure diagram

Table 1 Design parameters of bearings and rotors

\begin{tabular}{cc}
\hline Name & Value \\
\hline Bearing radius $R_{0}(\mu \mathrm{m})$ & 25 \\
Groove depth ratio $\mathrm{Hg}$ & 3.6 \\
Groove width ratio $\mathrm{Bg}$ & 0.4 \\
Groove number $\mathrm{Ng}$ & 6 \\
Spiral angle $\beta\left(^{\circ}\right)$ & 60 \\
Bearing clearance $h_{0}(\mu \mathrm{m})$ & 30 \\
Orifice diameter $d_{1}(\mathrm{~mm})$ & 0.2 \\
Number of gas supply holes & 2 \\
Number of hole $s$ & 12 \\
\hline
\end{tabular}
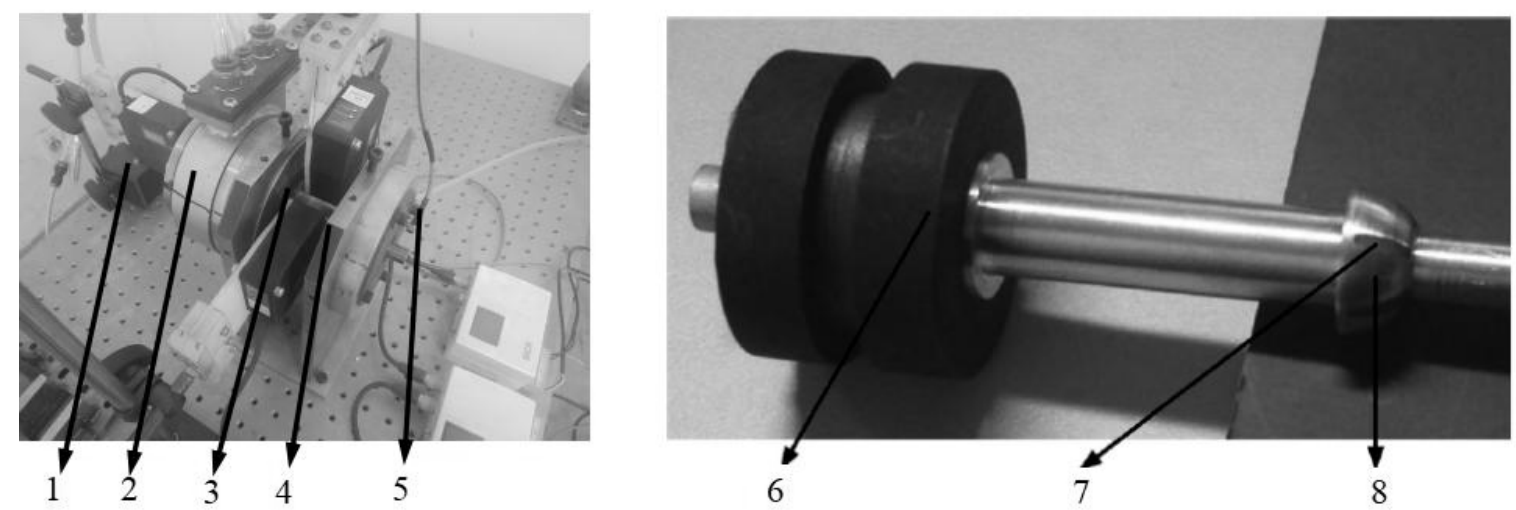

1-Laser displacement sensor; 2-Turbine; 3- Gas bearing-rotor system; 4- Frame ; 5- Speed sensor; 6-Gas bearing; 7-Spiral groove; 8-Rotor

Figure 6. Bearing test machine

\subsection{Experimental test system}

The experimental test system includes displacement detection, speed detection, and rotor testing. The German Sick ODM24-2 (s) laser displacement sensor measures the displacement signals of the rotor in the horizontal $(\mathrm{X})$, vertical $(\mathrm{Y})$, and axial $(\mathrm{Z})$ directions, 
as indicated in Figure 4. The Keyence FS-N10 fibr-optic speed sensor is used to measure the rotational speed signal at the rotor end face. A PCB board is used to design and construct the drive circuit of the rotor rubbing circuit, and the rubbing signal of the bearing and rotor is measured by the wiring terminal. HRsodft_DW_V2.06 acquisition software and the Shanghai Haonai 8-channel 16-bit data acquisition box are used to collect the data of the rotational speed, displacement, and rubbing. Combined with the MYSQL database, the system can carry out data acquisition and storage, with a resolution of $0.1 \mu \mathrm{m}$, displacement range of 24 to $26 \mathrm{~mm}$, speed range of 0 to $1800000 \mathrm{r} / \mathrm{min}$, and response frequency of $100 \mathrm{kHz}$, so that data can be analysed and processed online or offline.

\section{Gas bearing stability and dynamic characteristics testing experiment}

\subsection{Displacement calibration of initial test}

The valve is opened to supply gas to the bearing rotor at a pressure of $0.5 \mathrm{MPa}$. This status is maintained for $30 \mathrm{~s}$ to ensure that the rotor is running stably and no contamination occurs in the gas film gap. The bearing rotor is confirmed to be in a sound initial condition and the rotor gas supply system is closed.

The experimental procedure is as follows. The signal detection system is turned on, the sampling frequency is set to $6 \mathrm{kHz}$, the low-pass filter is set to $2 \mathrm{kHz}$, and signal sampling is started. The rotor horizontal and axial displacements $X_{0}$ and $Z_{0}$ are recorded, which are the initial rotor displacements in the horizontal and axial directions, respectively $\left(X_{0}=Z_{0}=0\right)$.The rotor vertical displacement data are recorded at the lowest vertical point $y_{0 \max }$; the rotor is manually raised to the highest point and the rotor vertical displacement data are recorded at the highest vertical point $y_{0 \text { min }}$. The initial displacement in the vertical direction is set as $y_{0}=1 / 2\left(y_{0 \min }+y_{0 \max }\right)$ and the rotor gap gas film thickness is set as $h_{0}=1 / 2\left(y_{0 \max }-y_{0 \min }\right)=2 e$.

\subsection{Experimental process}

The valve is opened to supply gas to the bearing rotor at a pressure of $0.5 \mathrm{MPa}$. This status is maintained for $30 \mathrm{~s}$ to ensure that the rotor is running stably and no contamination occurs in the gas film gap. The rotor gas supply pressure is adjusted to $0.3 \mathrm{MPa}$ and the rotor rotational speed is allowed to increase gradually and then operate stably at 5,000 $\mathrm{r} / \mathrm{min}$. The turbine gas supply system is turned on; firstly, the rotor speed stably increases in cycle 1. At this point, the rotor is operating in a linear stability state, and the rotor axis trajectory shape is an ellipse. During the initial stage, the rotor speed is low, and the gas film dynamic pressure effect and rotational inertia are small; the gas supply pressure fluctuation significantly influences the rotor operating state and the rotor axis trajectory line is blurry. As the rotational speed increases, the influence of the gas supply pressure fluctuation on the rotor operating state is reduced, and the power frequency vibration of the rotor caused by imbalance is strengthened. The rotor axis trajectory becomes clearer and the radius gradually increases; the rotor axis trajectory simultaneously maintains an elliptical shape when the rotational speed is approximately $10,000 \mathrm{r} / \mathrm{min}$. The rotor speed stably increases and reaches the first-order critical rotational speed at $17,456 \mathrm{r} / \mathrm{min}$. The rotational speed continues to rise and reaches the maximum speed of $35,000 \mathrm{r} / \mathrm{min}$ in cycle 
1, as illustrated in Figure 8-a. At this time, the power frequency vibration is $583 \mathrm{~Hz}$, which dominates; the rotor axis trajectory begins to diverge and an unstable trend appears. As the rotational speed continues to increase, the rotor enters cycle 2 operation at approximately $36,400 \mathrm{r} / \mathrm{min}$, as shown in Figure 8-b; the power frequency vibration is $606 \mathrm{~Hz}$ at this time, a half-frequency vortex appears, and the frequency is $303 \mathrm{~Hz}$. Additionally, the halffrequency vortex vibration amplitude increases rapidly, and the rotor axis trajectory changes to an " 8 " shape. The rotor exits from the linear stable state and enters the nonlinear stable state operation. The rotational speed increases further, following which the rotor enters the chaotic state at approximately 38,500 r/min and reaches the critical instability speed at $38,500 \mathrm{r} / \mathrm{min}$, as illustrated in Figure 8-c. A large amount of low frequencies and 1.5-fold frequency appear, the gas film oscillations intensify, and the rotor axis trajectory begins to degrade. The rotor exits the nonlinear stable state and the bearing is unstable. The rotor operates at the maximum rotational speed for $30 \mathrm{~s}$; thereafter, the turbine driving system is turned off, the rotor slows down to the initial state, sampling ends, and the experiment is complete.

According to the aforementioned procedure, the experiment is conducted again at rotor gas supply pressures of $0.1,0.2,0.4$, and $0.5 \mathrm{MPa}$.

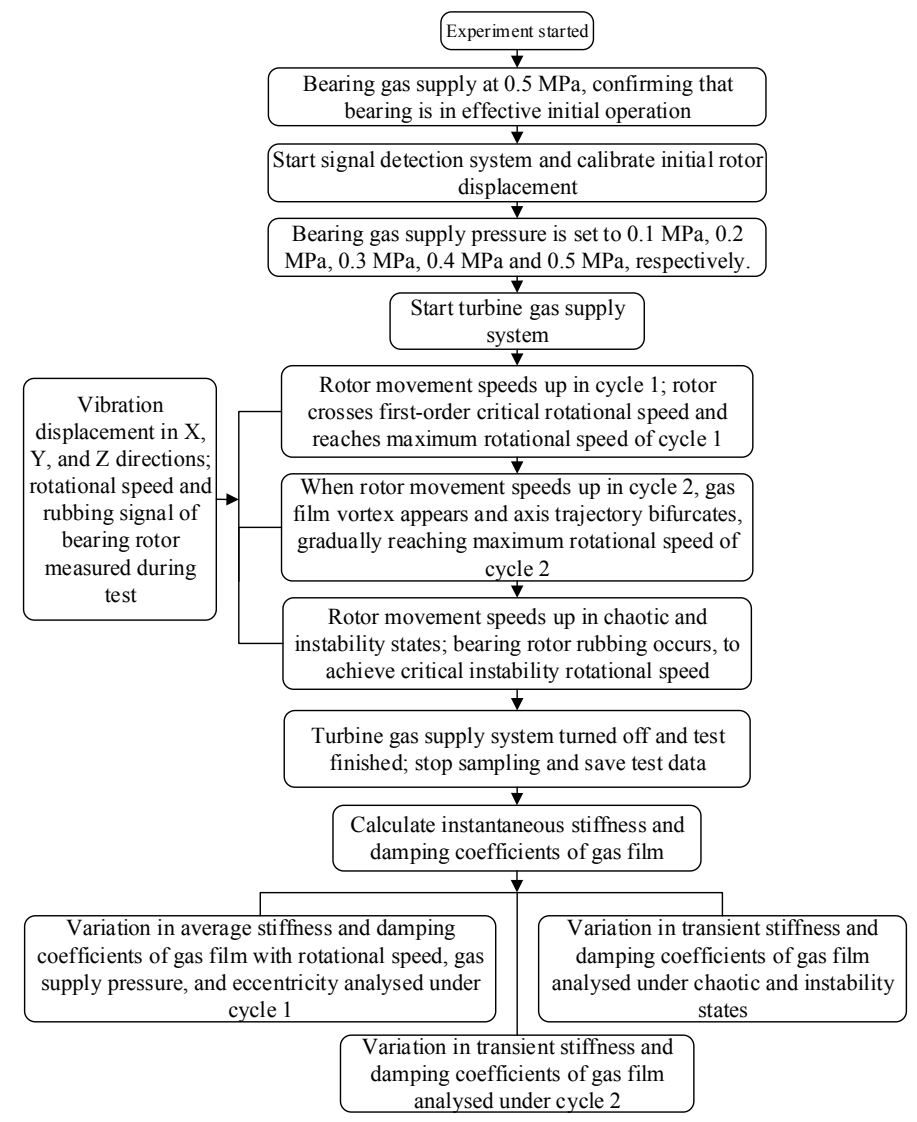

Figure 7. Experimental test flow chart 

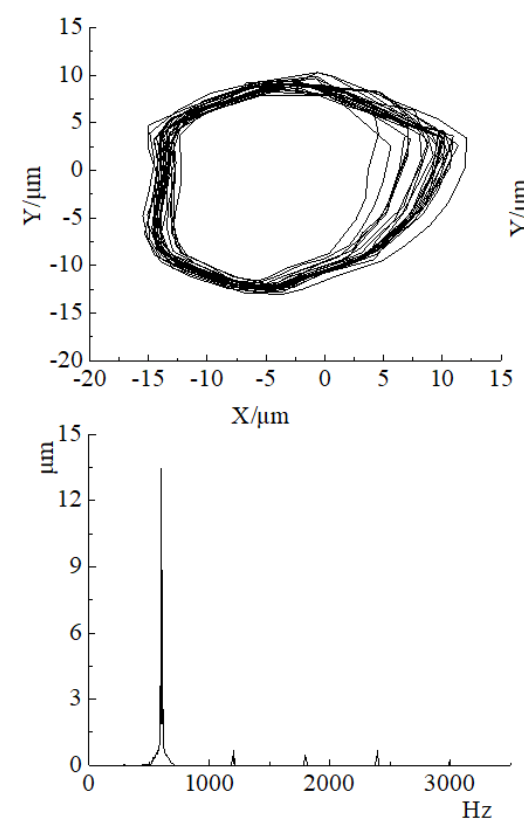

(a)Rotor speed 35000r/min
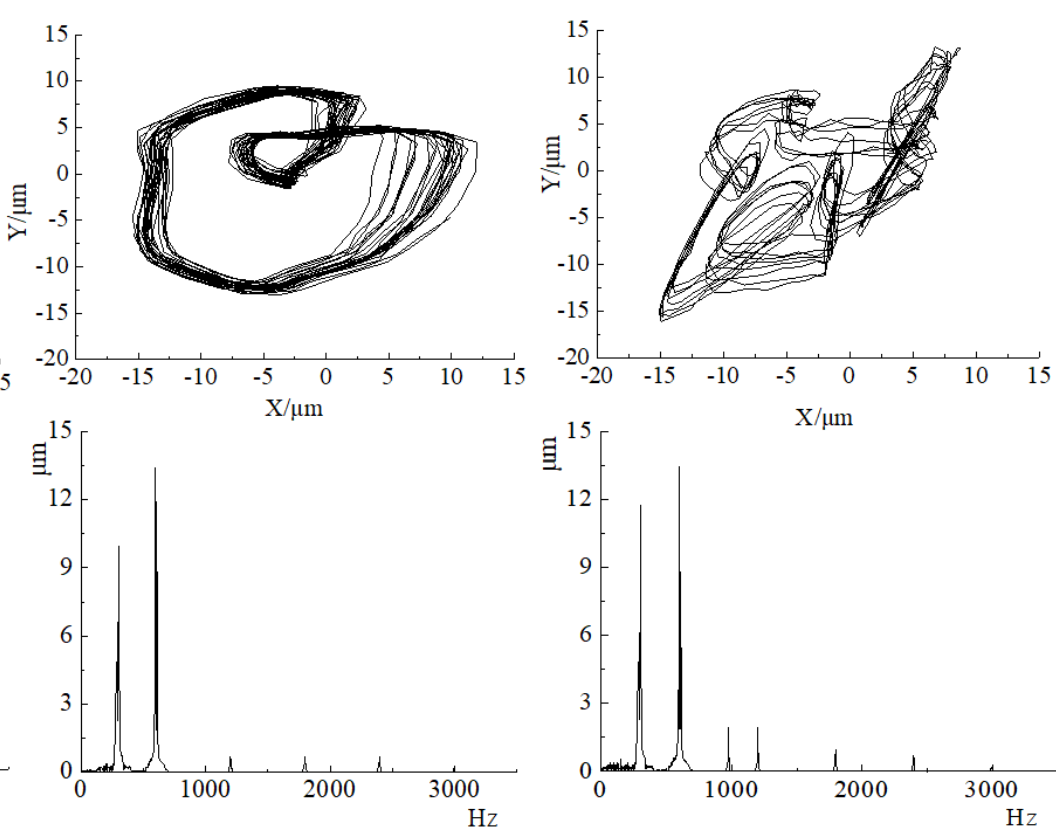

(b)Rotor speed 36400r/min

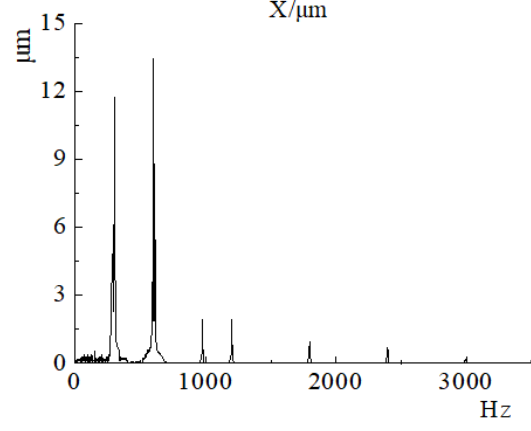

(c)Rotor speed 38500r/min

Figure 8. Axis trajectory diagram and spectrogram of different operating state of rotor

\section{Analysis of gas film operation dynamic characteristics}

\subsection{Cycle 1 gas film stiffness and damping variation pattern analysis}

Cycle 1 is the primary working region of the bearing ${ }^{(20)}$, in which the rotor is in the linear stable operation state. The gas film exhibits effective load capacity, the rotor vibration is small, and operation is smooth. Therefore, the experiment described in this section tests the impact of the rotational speed, gas supply pressure, and eccentricity on the gas film stiffness and damping coefficients.

To analyse the influence of rotational speed on gas film stiffness and damping, experimental data at rotational speeds of 10,000,15,000, 20,000, 25,000, and 30,000 r/min under a bearing gas supply pressure of $0.3 \mathrm{MPa}$ are extracted. The instantaneous stiffness and damping coefficients of the gas film are calculated and extracted when the eccentricity is 0.3 , and the average value is obtained. The variation in the gas film stiffness and damping coefficients with the rotational speed is illustrated in Figure 9.

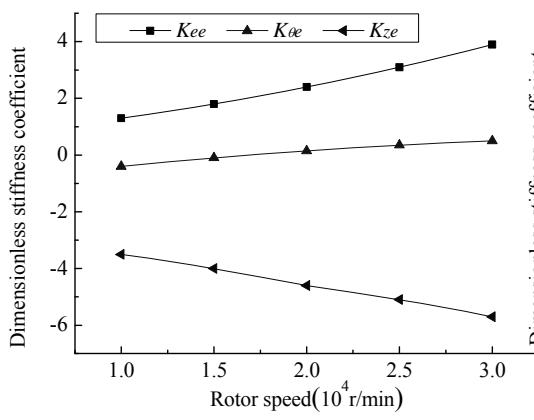

(a) $K_{e e}, K_{\theta e}, K_{z e}$

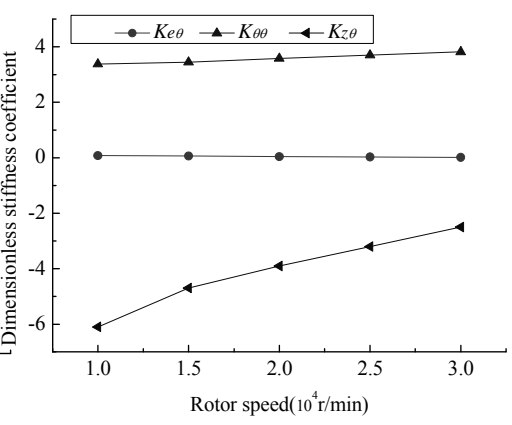

(b) $K_{e e}, K_{\theta \theta}, K_{z \theta}$

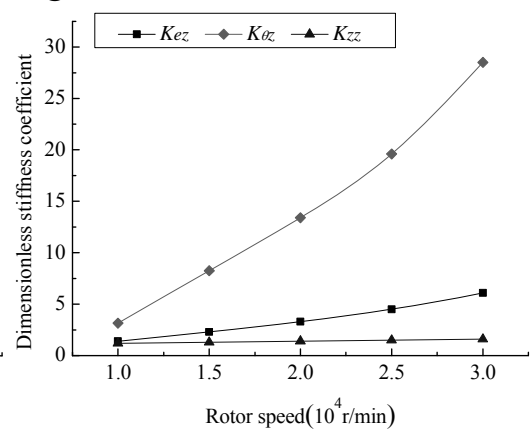

(c) $K_{e z}, K_{\theta z}, K_{\theta z z}$ 


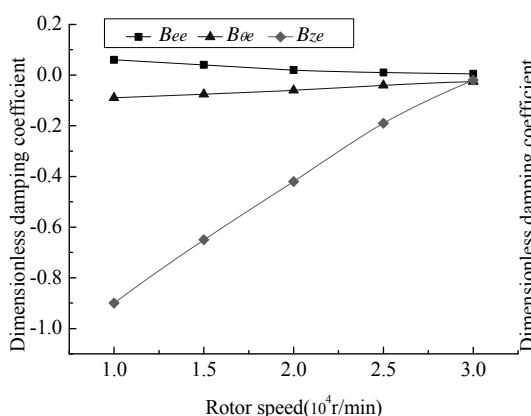

(d) $B_{e e}, B_{\theta e}, B_{z e}$

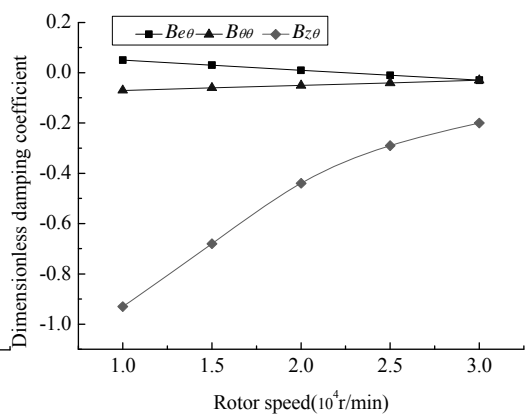

(e) $B_{e \theta}, B_{\theta \theta}, B_{z \theta}$

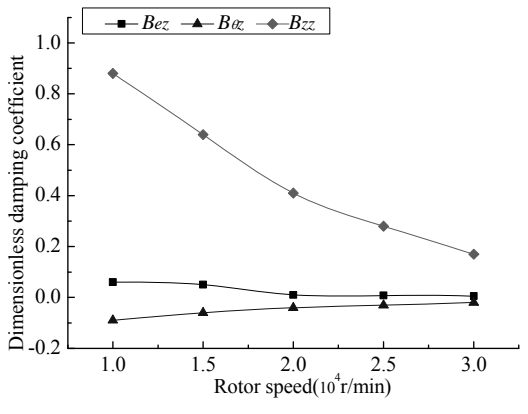

(f) $B_{e z}, B_{\theta z}, B_{z z}$

Figure 9.The relationship between the stiffness and damping coefficient of gas film and the

$$
\text { rotational speed }
$$

The relation between gas film stiffness and damping coefficient with rotational speed can be obtained by analyzing Figure 9:

(1) The stiffness coefficients $K_{e e}, K_{\theta e}, K_{z e}, K_{\theta \theta}, K_{z e}, K_{e z}$ and $K_{\theta z}$ increase as the rotational speed increases, and the increasing trends of $K_{e e}, K_{z e}, K_{e z}$ and $K_{\theta e}$ are clear. However, $K_{z \theta}$ decreases as the rotational speed increases, while $K_{e \theta}$ and $K_{z z}$ essentially remain unchanged. The damping coefficients gradually decrease as rotational speed increases, among which the decreasing trends of $B_{z e}, B_{z \theta}$, and $B_{z z}$ are more obvious. When the rotational speed reaches $30,000 \mathrm{r} / \mathrm{min}$, several damping coefficients decrease to close to zero.

(2) The variation law of the gas film stiffness and damping coefficients with rotational speed reveals that, as the rotational speed under a given gas supply pressure increases, the gas film dynamic pressure effect is enhanced, the load capacity increases, the stiffness increases, and the rotor rotational inertia and ability to restore itself to the original stable operation state under perturbation is improved. Furthermore, as the rotational speed increases, the damping coefficients decrease rapidly, causing the gas film vortex force to gradually become greater than the gas film damping force. The axis trajectory exhibits a gradual diverging trend, and the gas film stability is gradually reduced.

(3) The stiffness and damping coefficients related to the rotor trajectory amplitude change significantly, and the axis trajectory amplitude gradually increases. The change trends of the gas film stiffness and damping coefficients are the same in the radial plane and opposite in the axial direction; the rotor radial stability is greater than the axial stability. The variation magnitude of the trajectory shape relating to the cross-stiffness and damping constants $K_{e \theta}, K_{\theta e}, B_{\theta e}$ and $B_{e \theta}$ is small; therefore, the axis trajectory shape does not change significantly in cycle 1 , while the vibration amplitude increases gradually.

To analyse the influence of the gas supply pressure on the gas film stiffness and damping, experimental data at speeds of $20,000 \mathrm{r} / \mathrm{min}$ under bearing gas supply pressures of $0.1,0.2,0.3,0.4$, and $0.5 \mathrm{MPa}$ are extracted. The instantaneous stiffness and damping coefficients of the gas film are calculated and extracted when the eccentricity is 0.3 , and the average value is obtained. The variation in the gas film stiffness and damping coefficients with the gas supply pressure is illustrated in Figure 10. 


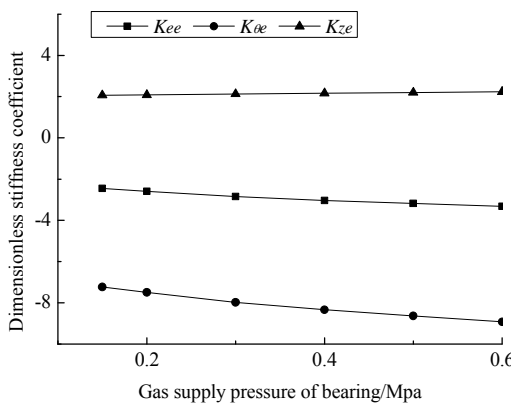

(a) $K_{e e}, K_{\theta z}, K_{z e}$

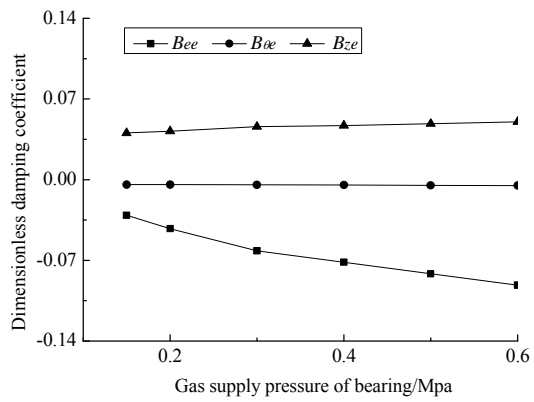

(d) $B_{e e}, B_{\theta e}, B_{z e}$

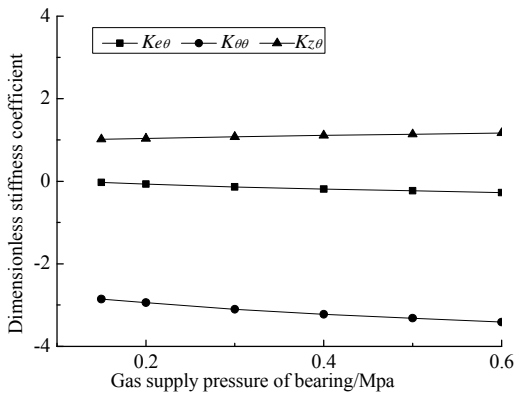

(b) $K_{e \theta}, K_{\theta \theta}, K_{z \theta}$

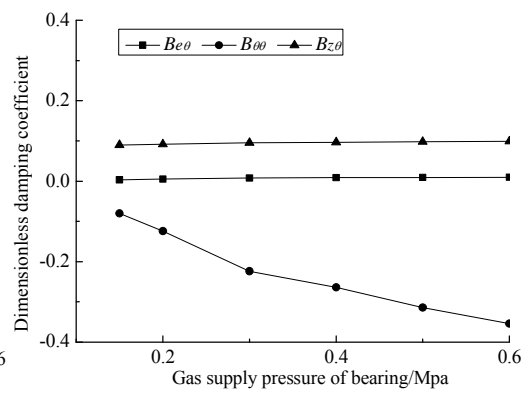

(e) $B_{e \theta}, B_{\theta \theta}, B_{z \theta}$

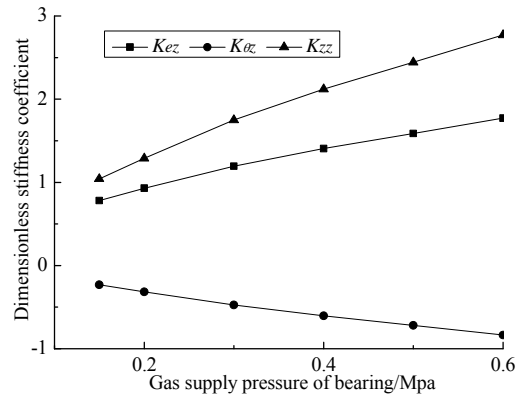

(c) $K_{e z}, K_{\theta z}, K_{z z}$

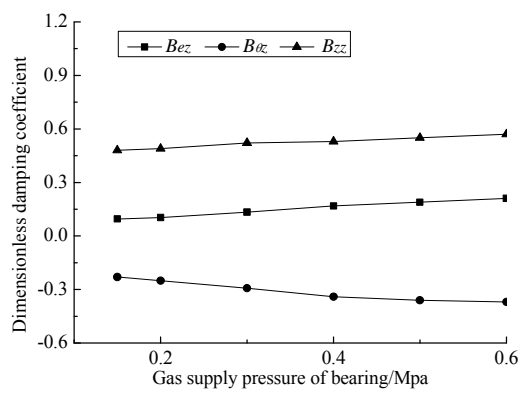

(f) $B_{e z}, B_{\theta z}, B_{z z}$

Figure 10. The relationship between the stiffness and damping coefficient of gas film and

bearing gas supply pressure

The relation between gas film stiffness and damping coefficient with bearing gas supply pressure can be obtained by analyzing Figure 10:

(1)Under a given eccentricity and rotational speed, when the bearing gas supply pressure increases, all of the gas film stiffness coefficients increase, among which the increasing trends of $K_{z e}, K_{z \theta}, K_{e z}, K_{\theta z}$ and $K_{z z}$ are clear. All of the damping coefficients increase as the bearing gas supply pressure increases, among which the increasing trends of $B_{z e}, B_{z \theta}, B_{e z}, B_{\theta z}, B_{\theta e}$ and $B_{z z}$ are clear.

(2) Under a given rotational speed and eccentricity, when the gas supply pressure increases, the gas film static pressure effect intensifies, the gas film vortex is reduced, and the oscillation is strengthened. Moreover, the load capacity of the gas film and its ability to resist external interference is intensified, and the gas film stiffness coefficients increase. When the rotor is disturbed, its ability to restore itself to the original stable state is enhanced, and the gas film damping coefficients increase. The rotor rotational inertia is large, the rotor radial stability is higher than the axial stability, and the gas film radial load capacity is higher than that in the axial direction. The gas film stiffness and damping coefficients $K_{z e}, K_{z \theta}, K_{e z}, K_{\theta z}, K_{z z}, B_{\theta z}$ and $B_{z z}$, related to the axial stability of the gas film, are significantly affected by the gas supply pressure.

(3) When the gas supply pressure increases, the gas film stiffness and damping coefficients increase, and the amount of vortex energy consumed by the damping force increases. The rotor axis trajectory exhibits a convergent trend, the gas film stability increases, and the rotor critical speed increases. Owing to the gas film dynamic and static pressure coupling effects and continuous energy input, the static pressure effect enhancement of the rotor orbit and radial stability is not obvious, while the axial load capacity and stability of the rotor are remarkably improved. Therefore, when the gas supply pressure during cycle 1 increases, the gas film load capacity and stability are enhanced, while the axis trajectory and vibration amplitude essentially remain unchanged. 
To analyse the influence of the rotor eccentricity on the gas film stiffness and damping, experimental data at rotational speeds of 20,000 r/min under a bearing gas supply pressure of $0.3 \mathrm{MPa}$ are extracted. The instantaneous stiffness and damping coefficients of the gas film are calculated and extracted when the rotor eccentricity is $0.1,0.2,0.3,0.4$, and 0.5 , and the average value is obtained. The variation in the gas film stiffness and damping coefficients with the eccentricity is illustrated in Figure 11.

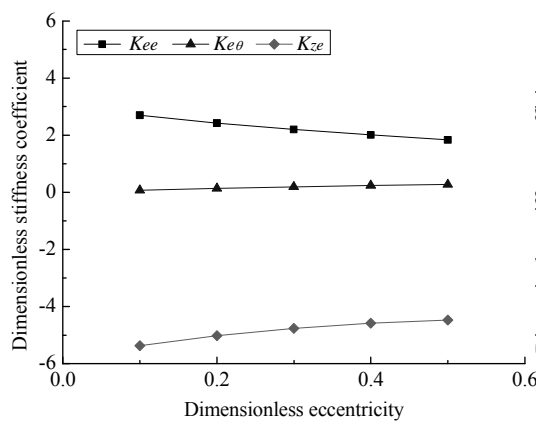

(a) $K_{e e}, K_{e \theta}, K_{\text {ze }}$

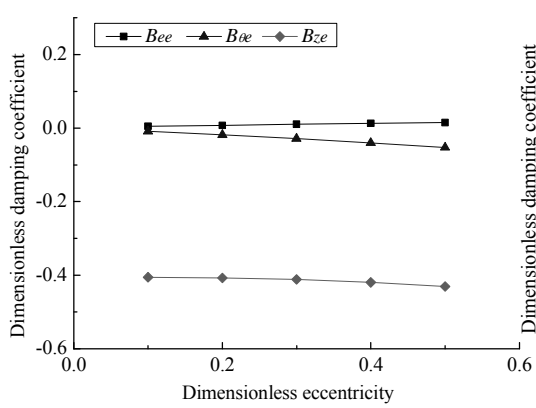

(d) $B_{e e}, B_{\theta e}, B_{z e}$

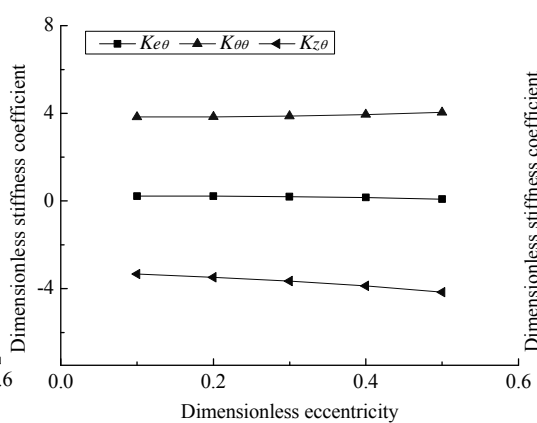

(b) $K_{e \theta}, K_{\theta \theta}, K_{z \theta}$

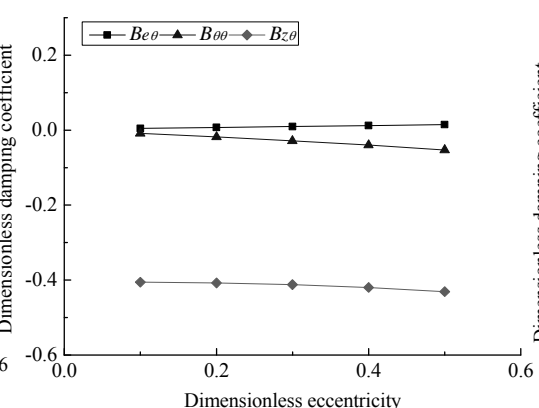

(e) $B_{e \theta}, B_{\theta \theta}, B_{z \theta}$

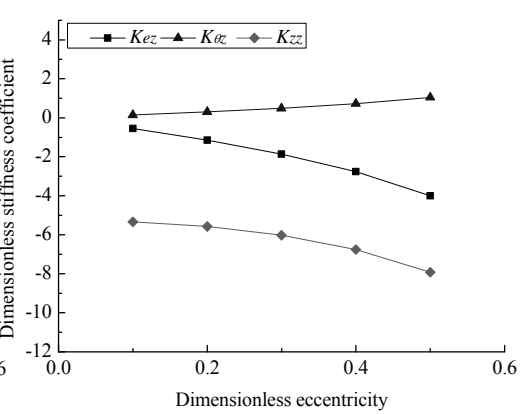

(c) $K_{e z}, K_{\theta z}, K_{z z}$

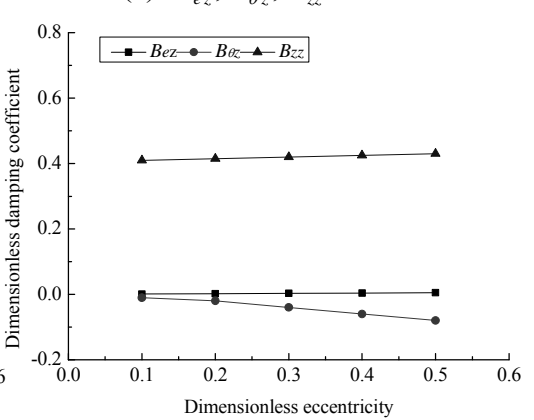

(f) $B_{e z}, B_{\theta z}, B_{z z}$

Figure 11. The relationship between gas film stiffness and damping coefficient and

\section{eccentricity}

The relation between gas film stiffness and damping coefficient with bearing gas supply pressure and eccentricity can be obtained by analyzing Figure 11:

(1) Under a given gas supply pressure and rotational speed, when the eccentricity increases, the gas film stiffness coefficients all increase, except for $K_{e e}$ and $K_{z e}$. The increasing trends of $K_{\theta \theta}, K_{e z}, K_{\theta z}$, and $K_{z z}$ are clear, while the increased amounts of $K_{\theta e}$, $K_{e \theta}$, and $K_{z \theta}$ are extremely small; $K_{e e}$ and $K_{z e}$ gradually decrease as the eccentricity increases. All of the damping coefficients increase as the eccentricity increases, among which the increasing trends of $B_{\theta e}, B_{\theta \theta}$, and $B_{z \theta}$ are clear.

(2) Under a given gas supply pressure and rotational speed, when the eccentricity increases, the gas film thickness decreases, the dynamic pressure effect is intensified, the load capacity increases, the stiffness and damping coefficients increase, and the gas film stability increases. The rotor rotational inertia is large, and its radial stability is higher than the axial stability; moreover, the gas film dynamic pressure effect in the radial direction is larger, and the gas film radial load capacity and stability are higher those that in the axial direction. The influence of eccentricity on the gas film axial stiffness and damping coefficients $K_{z e}, K_{z \theta}, K_{e z}, K_{\theta z}, K_{z z}, B_{z \theta}, B_{z e}, B_{\theta z}$ and $B_{z z}$ is significant.

(3) When gas film stiffness and damping coefficients increase, the amount of vortex energy consumed by the damping force increases; the rotor axis trajectory exhibits a convergent trend, and the gas film stability increases. However, when the rotational speed 
maintains a constant energy input, the rotor axis trajectory convergence is unclear. The variation magnitudes of the rotor axis trajectory-related gas film stiffness and damping coefficients $K_{e \theta}, K_{\theta e}, B_{e \theta}$, and $B_{\theta e}$ are small. Therefore, when the eccentricity increases during cycle 1 , the gas film load capacity and stability are enhanced, while the axis trajectory and vibration amplitude essentially remain unchanged.

\subsection{Cycle 2 gas film stiffness and damping variation pattern analysis}

During cycle 2 of the rotor operation, the gas film fluctuates, the rotor vibrations intensify, a half-frequency vortex appears, which induces gas film oscillation, and the gas film stability is reduced. The rotor is operating in the nonlinear stable operation state. If external interference or energy input is injected at this point, the rotor will enter a chaotic, unstable operating state. Rotor cycle 2 is the limit state of the stable rotor operation. Therefore, the dynamic characteristics of the gas film under the rotor cycle 2 operation are studied experimentally in this section.

To analyse the change rule of the gas film instantaneous stiffness and damping in cycle 2 of the rotor operation, experimental rotor data at speeds of $36,400 \mathrm{r} / \mathrm{min}$ under a bearing gas supply pressure of $0.3 \mathrm{MPa}$ are extracted. The data processing system extracts the instantaneous stiffness and instantaneous damping coefficients in 20 sampling processes at an eccentricity of 0.3. The orders of the gas film stiffness and damping coefficients with similar values are readjusted. The variation law of the instantaneous stiffness and damping coefficients of the gas film is illustrated in Figure 12.

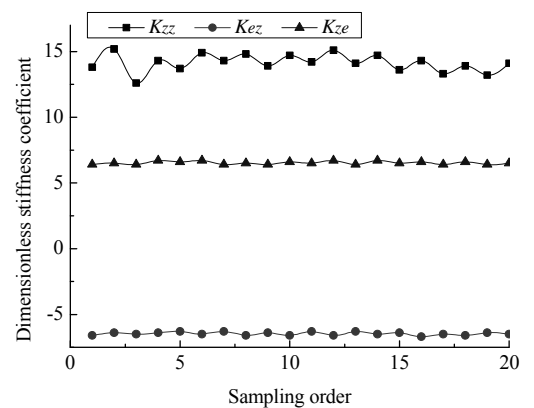

(a) $K_{z z}, K_{e z}, K_{z e}$

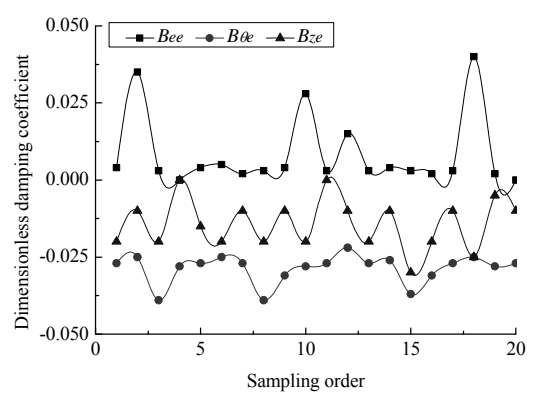

(d) $B_{e e}, B_{\theta e}, B_{z e}$

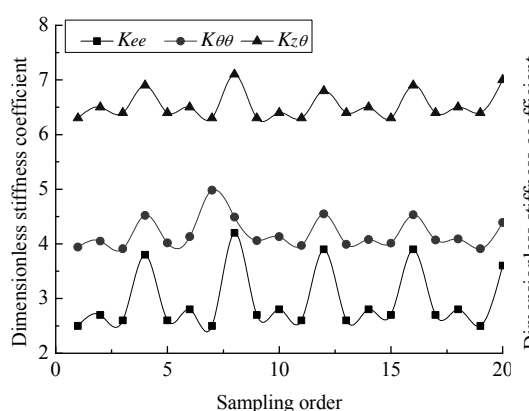

(b) $K_{e e}, K_{\theta \theta}, K_{z \theta}$

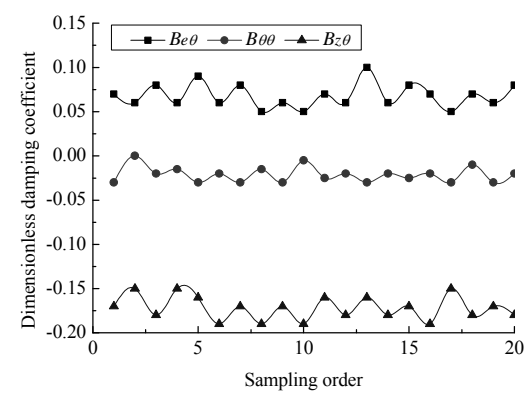

(e) $B_{e \theta}, B_{\theta \theta}, B_{z \theta}$

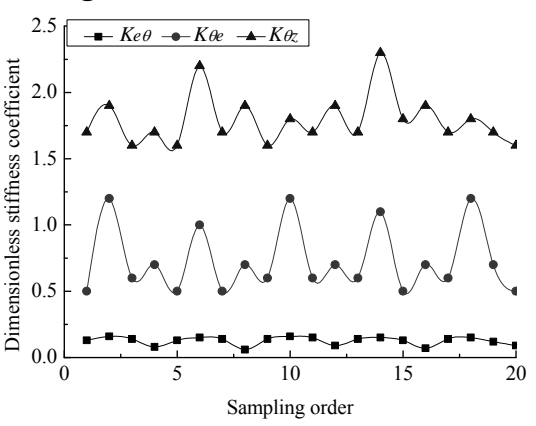

(c) $K_{e \theta}, K_{\theta e}, K_{\theta z}$

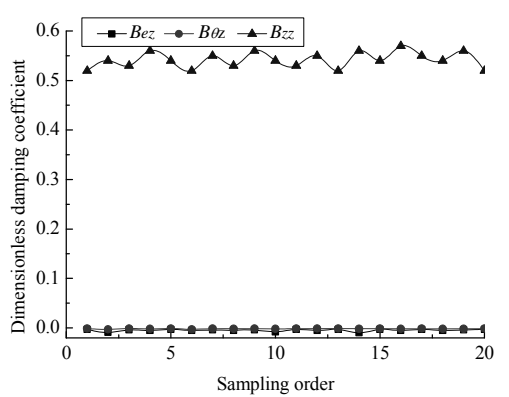

(f) $B_{e z}, B_{\theta z}, B_{z z}$

Figure 12. Change law of real time stiffness and damping coefficient of cycle 2

The relation between gas film instantaneous stiffness and instantaneous damping coefficient with the sequence order can be obtained by analyzing Figure12:

(1) When the rotor is operating in cycle 2, the gas film instantaneous stiffness coefficient values exhibit a fluctuation of approximately 2 times, among which the oscillation amplitudes of $K_{e e}, K_{\theta \theta}, K_{z \theta}, K_{e \theta}, K_{\theta e}$ and $K_{\theta z}$ are larger. Moreover, the gas 
film instantaneous damping coefficient values exhibit a fluctuation of approximately 2 to 5 times, among which the oscillation amplitudes of $B_{e e}, B_{\theta e}, B_{z e}, B_{e \theta}, B_{\theta \theta}$ and $B_{z \theta}$ are larger.

(2) Compared to cycle 1, the dynamic pressure effect and gas film force fluctuation increase in cycle 2. A half-frequency vortex appears, which induces gas film oscillation. The gas film instantaneous stiffness and damping coefficient values exhibit vibrations; the rotor vibrates near the static equilibrium point, and the gas film stability is reduced. The damping force is not sufficient to eliminate the gas film vortex energy; the rotor axis trajectory gradually diverges and the vibration amplitude increases, while the rotor axis trajectory is changed to an " 8 " shape.

(3) The rotor vibration induced by the gas film oscillation and non-equilibrium is primarily concentrated in the radial direction. The stiffness and damping coefficients $K_{e e}$, $K_{\theta \theta}, K_{z \theta}, K_{e \theta}, K_{\theta e}, K_{\theta z}, B_{e e}, B_{\theta e}, B_{z e}, B_{e \theta}, B_{\theta \theta}$ and $B_{z \theta}$, associated with the radial trajectory shape exhibit large fluctuation amplitudes, and the rotor radial stability is reduced at a faster rate and greater amplitude than the axial stability. The stiffness and damping coefficient vibration can be used as a judgement sign prior to rotor instability; the vibration amplitude is related to the gas film force fluctuation.

\subsection{Chaos and instability time of rotor gas film stiffness and damping variation}

\section{pattern analysis}

When the rotor is running in the chaos state, 1.5 times the frequency and a substantial amount of low frequencies appear, the force fluctuation and stability of the gas film increase, the rotor will be unstable, and rubbing of the bearing rotor appears. This section analyses the dynamic characteristics of the gas film when the rotor is in the chaotic or unstable operation state and explores the forecasting method and film stability control of the rotor operation state.

To analyse the change rule of the gas film instantaneous stiffness and damping when the rotor enters the chaotic or unstable operation state, experimental rotor data at speeds of $38,500 \mathrm{r} / \mathrm{min}$ under a bearing gas supply pressure of $0.3 \mathrm{MPa}$ are extracted. The data processing system extracts the instantaneous stiffness and damping coefficients in 20 sampling processes at an eccentricity of 0.5 . The orders of the gas film stiffness and damping coefficients with similar values are readjusted. The variation law of the instantaneous stiffness and damping coefficients of the gas film is illustrated in Figure 13.

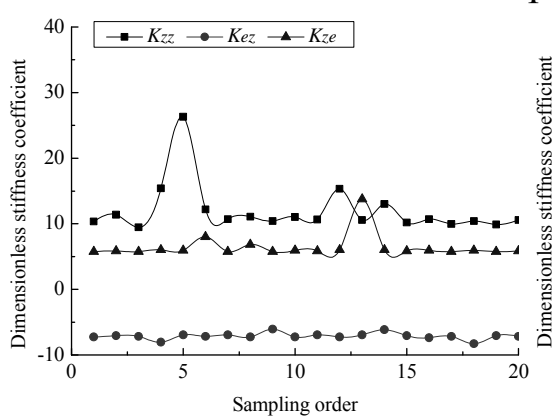

(a) $K_{z z}, K_{e z}, K_{z e}$

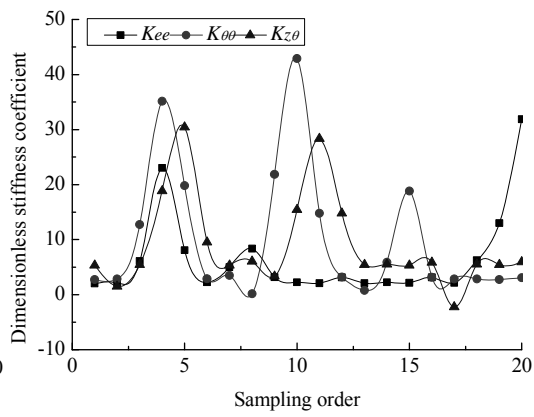

(b) $K_{e e}, K_{\theta \theta}, K_{z \theta}$

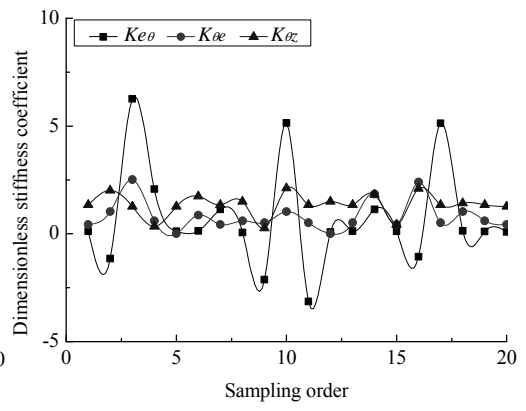

(c) $K_{e \theta}, K_{\theta e}, K_{\theta z}$ 


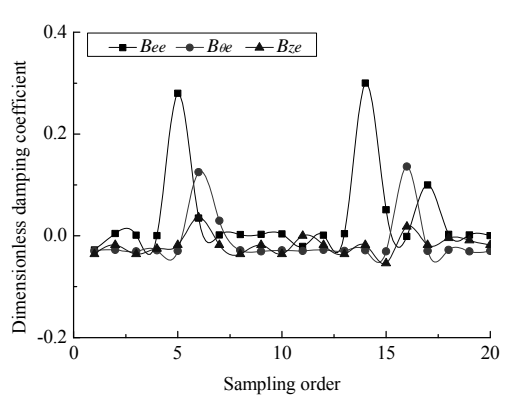

(d) $B_{e e}, B_{\theta e}, B_{z e}$

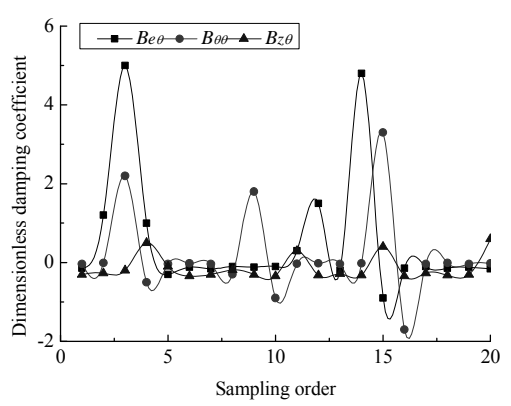

(e) $B_{e \theta}, B_{\theta \theta}, B_{z \theta}$

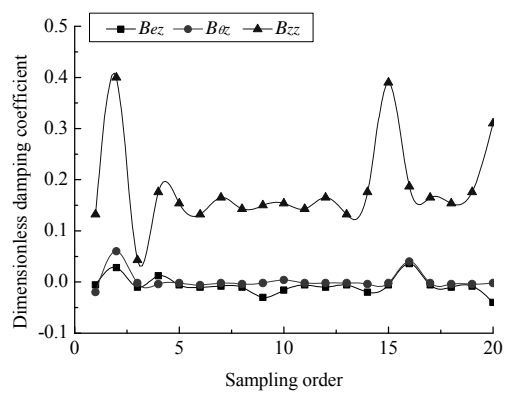

(f) $B_{e z}, B_{\theta z}, B_{z z}$

Figure 13. The gas film rotor instability of real time stiffness and damping coefficient of variation

Figure 13 analysis bearing rotor instability when real-time film stiffness and damping coefficients of the rule of variation:

(1) When the rotor enters the chaotic or unstable operation state, if rubbing appears, the gas film instantaneous stiffness coefficient value increases by more than 10-fold, among which the change degrees in the values of $K_{e e}, K_{\theta \theta}, K_{\theta e}$ and $K_{e \theta}$ are significant. The gas film instantaneous damping coefficient values increase by more than 25 -fold, among which the change degrees in the values of $B_{e e}, B_{\theta \theta}, B_{e \theta}$ and $B_{\theta e}$ are significant, and stiffness and damping coefficient distortion appears.

(2) In contrast to cycle 2, when the rotor is in the chaotic or unstable operation state, the gas viscosity decreases, 1.5 times the frequency and substantial amounts of low frequencies appear, the gas film force fluctuation increases, and the steady state bearing capacity decreases. Moreover, the stiffness and damping coefficients decrease, the gas film stability decreases, the energy consumed by the damping force is reduced, and the gas film vortex energy increases rapidly. Uncontrollable vibration of the rotor appears near the static equilibrium position, the rotor axis trajectory diverges and the amplitude increases, and rubbing of the bearing rotor appears.

(3) At the rubbing point, the bearing and rotor are directly and rigidly in contact; the instantaneous force of the bearing on the rotor increases suddenly, and the rotor operation state changes. Macroscopically, the gas film instantaneous stiffness and damping coefficients simultaneously increase in an instant and distortion occurs. In a few instances, only the gas film stiffness coefficients exhibit distortion at the rubbing moment, and the damping coefficients are missing. Rubbing mainly occurs in the radial direction, and the stiffness damping coefficients $K_{e e}, K_{\theta \theta}, K_{e \theta}, K_{\theta e}, B_{e e}, B_{\theta \theta}, B_{e \theta}$ and $B_{\theta e}$ related to the radial rubbing, exhibit large distortion amplitudes and greater distortion times. The distortion degree and density of the stiffness damping coefficients are related to whether the rotor is completely rubbed. The gas film stiffness and damping coefficient distortion can be used as a sign of rotor instability or failure.

\section{Conclusions}

(1) The mechanical model of the gas film was analysed for the lubrication characteristics of spherical spiral groove hybrid gas bearings. The relationship between the gas film force increment and the displacement and velocity disturbances was established. Combined with the bearing rotor motion equation, the solving equations for the gas film stiffness and damping coefficients were established. 
(2) Experimental data of the rotational speed and vibration displacement under different rotor operation states were measured on a gas bearing tester. The rolling iterative method was used to input the pre-processed results into a MATLAB solving routine to calculate the instantaneous gas film stiffness and damping coefficients; simultaneously, the bearing rotor operation process and state variation were analysed. The proposed method combines gas lubrication theory with the mechanical model and includes collaboration with experimental identification and testing, which can effectively obtain the dynamic characteristics of the gas film during the gas bearing operation.

(3) The rotor is in the linear stable operation state in cycle 1. When increasing the rotor speed, the dynamic and static pressure coupling effects increase, and the stability of the gas film is decreased by the change in dynamic characteristics. The interaction between the gas film stiffness and damping can enhance the bearing stability. The gas supply pressure exerted on the bearing increases, the static pressure effect is enhanced, the bearing capacity of the gas film increases, the vortex of the gas film decreases, and the gas film oscillation increases. Moreover, the stability of the gas film increases; the influence of the rotor speed and eccentricity on the radial stability of the gas film and the influence of the pressure exerted on the bearing on the rotor axial stability are significant.

(4) From the analysis of the rotor mechanical behaviour, it is revealed that the influence of the rotor rotational inertia and the gyro effect on the rotor radial stability is greater than that on the axial stability. When the rotational speed is increased in cycle 1, the power frequency vibration owing to non-equilibrium increases, and the gas film dynamic pressure effect is intensified. In cycle 2 of the rotor operation, gas film fluctuation appears, which causes gas film stiffness and damping coefficient vibrations. A halffrequency vortex appears, which induces gas film oscillation; the rotor enters the nonlinear stable operation state and the gas film stability is reduced. When the rotor operates in the chaotic and unstable states, the gas film force fluctuation and gas film oscillation are intensified. The rotor exhibits uncontrollable vibration near the static equilibrium position. The gas film stability is further degraded, the bearing and rotor exhibit a rubbing phenomenon, and the gas film instantaneous stiffness and damping coefficients exhibit distortion. Thus, the rotor operation state can be evaluated and predicted according to the variation characteristics of the gas film stiffness and damping coefficients.

\section{Acknowledgement}

This work is supported by National Natural Science Foundation of China (Grant no: 51475142), and Program for Innovative Research Team (in Science and Technology) in University of Henan Province (Grant no: 13IRTSTHN025).

\section{References}

1. Liu R and Wang X. Dynamic characteristics analysis of micro air spiral grooved thrust bearing-rotor system. 6th IEEE International Conference on Nano/Micro Engineered and Molecular Systems, NEMS 2011, Kaohsiung, Taiwan, February 20 February 23, 2011, pp. 719-723.

2. Cui DP, Yao YX and Qin DL. Study on the dynamic characteristics of a new type externally pressurized spherical gas bearing with slot-orifice double restrictors. Tribol Int. 2010; 43: 822-830.

3. Zhang XQ, Wang XL, Si LN, Liu YD, Shi WT and Xiong GJ. Effects of temperature on nonlinear dynamic behavior of gas journal bearing-rotor systems for microengine. Tribol Trans. 2016; 59: 944-956. 
4. Jia $\mathrm{CH}$, Pang $\mathrm{HJ}$, Ma WS, et al. Analysis of dynamic characteristics and stability prediction of gas bearings. Ind Lubr Tribol 2017; 69:123-130.

5. Li SL. Measurement of rigzdity of about the aerostatic bearing for precision centrifuge use. Journal of Harbin Institute of Technology. 2002; 34: 797-800.

6. Zhang WM, Zhou JB and Meng G. Performance and stability analysis of gaslubricated journal bearings in MEMS. Tribol Int. 2011; 44: 887-897.

7. Jia $\mathrm{CH}$, Pang HJ, Ma WS, et al. Dynamic stability prediction of spherical spiral groove hybrid gas bearings rotor system. J Tribol-T ASME 2016; 139:021701-021713.

8. Du JJ, Yang GW, Ge WP and Liu T. Nonlinear dynamic analysis of a rigid rotor supported by a spiral-grooved opposed-hemisphere gas bearing. Tribol Trans. 2016; 59: 781-800.

9. Du JJ, Zhang GQ, Liu T and To S. Improvement on load performance of externally pressurized gas journal bearings by opening pressure-equalizing grooves. Tribol Int. 2014; 73: 156-166.

10. Belforte $\mathrm{G}$, Raparelli $\mathrm{T}$ and Viktorov V. Modeling and identification of gas journal bearings: Self-acting gas bearing results. J Tribol-Trans ASME. 2002; 124: 716-724.

11. Guo LB and Wang ZW. New method of dynamic test for static stiffness of externally pressurized gas bearing. Chinese Journal of Mechanical Engineering. 2007; 43: 21-26.

12. Sim K, Lee YB and Kim TH. Effects of mechanical preload and bearing clearance on rotordynamic performance of lobed gas foil bearings for oil-free turbochargers. Tribol Trans. 2013; 56: 224-235.

13. Sim K, Lee YB and Kim TH. Rotordynamic analysis of an oil-free turbocharger supported on lobed gas foil bearings-predictions versus test data. Tribol Trans. 2014; 57: 1086-1095.

14. Yang LH, Shi JH, Liu H and Yu L. Experimental study on dynamic performance of compliant aerodynamic foil journal air bearings. Tribology. 2006; 26: 353-357.

15. Delgado A. Experimental identification of dynamic force coefficients for a $110 \mathrm{~mm}$ compliantly damped hybrid gas bearing. J Eng Gas Turbines Power-Trans ASME. 2015; 137: V07BT32A025, 1-9.

16. Ma B, Sun W, Lai TW, Zheng YQ, Chen ST and Hou Y. Experimental study on gas lubricated hydrodynamic bump type foil bearing. Journal of Xi'an Jiaotong University. 2014; 48: 118-122.

17. Jia $\mathrm{CH}$, Pang $\mathrm{HJ}$ and Qiu M. Analysis of dynamic characteristics and stability prediction of spherical spiral groove hybrid gas bearings. Journal of Aerospace Power. 2017; 32: 1400-1411.

18. Pierart FG and Santos IF. Active lubrication applied to radial gas journal bearings. Part 2: Modelling improvement and experimental validation. Tribol Int. 2016; 96: 237-246.

19. Xia XT, Ye L, Li YF and Chang Z. Reliability evaluation based on hierarchical bootstrap maximum entropy method. Binggong Xuebao/Acta Armamentarii. 2016; 37: 1317-1329.

20. Wang CC and Kuo CL. Nonlinear dynamic analysis of a relatively short spherical gas journal bearing system. Journal of Mechanical Science and Technology. 2010; 24: $1565-1571$. 
2019-01-15

Study on dynamic characteristics of gas

films of spherical spiral groove hybrid

gas bearings

Jia, Chenhui

SAGE

Chenhui Jia, Haijiang Zhang, Shijun Guo, et al., Study on dynamic characteristics of gas films of spherical spiral groove hybrid gas bearings. Proceedings of the Institution of Mechanical

Engineers, Part J: Journal of Engineering Tribology, Available online 15 January 2019

https://doi.org/10.1177/1350650118823892

Downloaded from Cranfield Library Services E-Repository 